\title{
Concentration of low energy extremals
}

by

\section{FLUCHER}

GFAI AG, Banking systems, Gludz-Blotzheimerstr. 1, $\mathrm{CH}-4503$ Solothurn, Switzerland, mfl@gfai.ch

and

\section{S. MÜLLER}

Max-Planck Institut für Mathematik

in den Naturwissenschaften, Inselstr. 22-26, D-04103 Leipzig, Germany

Stefan.Mueller@mis.mpg.de

ABSTRACT. - We study variational problems of the form

$$
\sup \left\{\int_{\Omega} F(u): \int_{\Omega}|\nabla u|^{2} \leq \varepsilon^{2}, u=0 \text { on } \partial \Omega\right\}
$$

with small $\varepsilon$ and $0 \leq F(t) \leq c|t|^{\frac{2 n}{n-2}}$ on a domain of dimension $n \geq 3$. The corresponding Euler Lagrange equation is a semilinear Dirichlet problem

$$
\begin{aligned}
-\Delta u & =\lambda f(u) \text { in } \Omega, \\
u & =0 \text { on } \partial \Omega
\end{aligned}
$$

with $f=F^{\prime}$ and a large Lagrange multiplier $\lambda$. Our goal is to obtain qualitative information on the extremals $u_{\varepsilon}$ for small $\varepsilon$. The integrand $F$ can be nonconvex and discontinuous. Thus our results apply to nonlinear eigenvalue problems as well as to certain free-boundary problems.

Our starting point is a generalized Sobolev inequality that covers the classical Sobolev inequality and the isoperimetric inequality relating capacity and volume as special cases. Using a local version of this inequality

Mathematical Subjects Classification: 35J20, 35B40

Annales de l'Institut Henri Poincaré - Analyse non linéaire - 0294-1449 
we prove a generalized concentration-compactness alternative and show that as $\varepsilon \rightarrow 0$ the extremals concentrate at a single point. The local behaviour of the extremals near the concentration point depends only on $F$. On a microscopic scale they tend to an extremal for the generalized Sobolev constant on $\mathbb{R}^{\mathrm{n}}$ provided that $F$ satisfies certain growth conditions at 0 and infinity.

(C) 1999 L'Association Publications de l'Institut Henri Poincaré. Published by Elsevier B.V. All rights reserved

Key words: variational problem, concentration, critical Sobolev exponent, free-boundary problem.

RÉSUMÉ. - Nous étudions des problèmes variationnels

$$
\sup \left\{\int_{\Omega} F(u): \int_{\Omega}|\nabla u|^{2} \leq \varepsilon^{2}, u=0 \text { sur } \partial \Omega\right\}
$$

où $\varepsilon$ est petit et $0 \leq F(t) \leq c|t|^{\frac{2 n}{n-2}}$ et $\Omega$ est un domaine de dimension $n \geq 3$. L'équation d'Euler-Lagrange correspondante est un problème de Dirichlet semilinéaire

$$
\begin{aligned}
-\Delta u & =\lambda f(u) \text { in } \Omega \\
u & =0 \text { on } \partial \Omega
\end{aligned}
$$

où $f=F^{\prime}$ et $\lambda$ est un multiplicateur de Lagrange grand. Notre objectif est d'obtenir des informations qualitatives concernant les solutions extrémales $u_{\varepsilon}$ pour $\varepsilon$ petit. La fonction $F$ peut être non convexe et discontinue.

Nos résultats s'appliquent par conséquent à des problèmes aux valeurs propres non linéaires et à certains problèmes à frontière libre. Notre point de départ est une inégalité de Sobolev généralisée qui contient l'inégalité de Sobolev classique et l'inégalité isopérimétrique qui relie la capacité et le volume. En utilisant une version locale de cette inégalité nous démontrons une version généralisée de l'alternative concentration-compacitée de Lions et nous montrons que les solutions extrémales se concentrent en un seul point lorsque $\varepsilon \rightarrow 0$. Le comportement local de ces solutions extrémales au voisinage du point de concentration dépend uniquement de $F$. A une échelle microscopique elle tend vers la solution extrémale de la constante de Sobolev généralisée dans $\mathbb{R}^{\mathfrak{n}}$ à condition que $F$ vérifie certaines conditions d'accroissement en zéro et à l'infini.

(C) 1999 L'Association Publications de l'Institut Henri Poincaré. Published by Elsevier B.V. All rights reserved

Mots clés : problème variationnel, concentration, exposant de Sobolev critique, problème à frontière libre. 


\section{INTRODUCTION}

We investigate the asymptotic behaviour of solutions and approximate solutions $u_{\varepsilon}$ of the variational problem

$$
\sup \left\{\int_{\Omega} F(u): \int_{\Omega}|\nabla u|^{2} \leq \varepsilon^{2}, u=0 \text { on } \partial \Omega\right\}
$$

in the limit $\varepsilon \rightarrow 0$. The integrand $F$ is supposed to satisfy the growth condition

$$
0 \leq F(t) \leq c|t|^{2^{*}},
$$

where $2^{*}:=\frac{2 n}{n-2}$ denotes the critical Sobolev exponent. For smooth $F$ the extremals $u_{\varepsilon}$ satisfy the Euler Lagrange equation

$$
\begin{aligned}
-\Delta u_{\varepsilon} & =\lambda_{\varepsilon} f\left(u_{\varepsilon}\right) \text { in } \Omega \\
u_{\varepsilon} & =0 \text { on } \partial \Omega
\end{aligned}
$$

with $f=F^{\prime}$. Our main result, Theorem 3, says that:

1. The extremals $\left(u_{\varepsilon}\right)$ concentrate at a single point $x_{0} \in \bar{\Omega}$.

2. On a microscopic scale near the concentration point they tend to an entire extremal, i.e. to a solution of (1) on $\mathbb{R}^{\mathbf{n}}$ with $\varepsilon=1$.

The first result extends work by P.L. Lions for the critical power function to general integrands. The second one is essentially contained in Lions [10]. Nevertheless we present a new proof based on a local generalized Sobolev inequality (Corollary 9) that is used several times for both results and should be useful for other purposes as well. In a forthcoming paper [5] we discuss the problem of identifying the concentration point $x_{0}$.

\section{DEFINITIONS AND HYPOTHESES}

1. In this paper we consider the so called it zero mass case characterized by $f(0)=0$. In this case the natural function space for variational problems of the form (1) is $D^{1,2}(\Omega)$, defined as the closure of $C_{c}^{\infty}(\Omega)$ with respect to the norm

$$
\|\nabla v\|_{2}:=\left(\int_{\Omega}|\nabla v|^{2}\right)^{1 / 2}
$$

For the positive mass case with $f(0)<0$ the appropriate space is the usual Sobolev space $H_{0}^{1}(\Omega)$. The only constant function in $D^{1,2}(\Omega)$ 
is 0 . If $\Omega$ has finite volume $D^{1,2}(\Omega)$ and $H_{0}^{1}(\Omega)$ are the same spaces. In general, however, $D^{1,2}(\Omega) \not \subset L^{2}(\Omega)$. For instance the functions $v(x)=\min \left(1,|x|^{-\alpha}\right)$ with $(n-2) / 2<\alpha \leq n / 2$ are contained in $D^{1,2}\left(\mathbb{R}^{\mathrm{n}}\right)$ but not in $L^{2}\left(\mathbb{R}^{\mathrm{n}}\right)$. Throughout this paper functions in $D^{1,2}(\Omega)$ are extended by 0 to all of $\mathbb{R}^{\mathrm{n}}$. Thus $D^{1,2}(\Omega) \subset D^{1,2}\left(\mathbb{R}^{\mathrm{n}}\right)$. Weak convergence, denoted as

$$
v_{\varepsilon} \rightarrow v \text { in } D^{1,2}(\Omega)
$$

is equivalent to

$$
\nabla v_{\varepsilon} \rightarrow \nabla v \text { weakly in } L^{2}(\Omega)
$$

which implies

$$
\begin{aligned}
& v_{\varepsilon} \rightarrow v \text { weakly in } L^{2^{*}}(\Omega), \\
& v_{\varepsilon} \rightarrow v \text { in } L_{\text {loc }}^{p}(\Omega)
\end{aligned}
$$

for every $p<2^{*}$ by Sobolev's embedding theorem and Rellich's compactness theorem.

2. We consider the variational problem (1) for integrands $F$ satisfying $F^{\prime}(t) \geq F^{\prime}(0)$. Without loss of generality we can assume $F^{\prime}(0)=0$. To motivate our hypotheses on $F$ recall Sobolev's inequality

$$
\int_{\Omega}|v|^{2^{*}} \leq S^{*}\left(\int_{\Omega}|\nabla v|^{2}\right)^{\frac{n}{n-2}}
$$

for functions in $D^{1,2}(\Omega)$. The optimal constant $S^{*}$ is independent of $\Omega$. Usually

$$
S_{n}=\left(S^{*}\right)^{\frac{2-n}{n}}=n(n-2) \pi\left(\frac{\Gamma(n / 2)}{\Gamma(n)}\right)^{2 / n}
$$

is called the best Sobolev constant. Throughout this paper we make the following general hypotheses.

$(\Omega) \Omega$ is a domain in $\mathbb{R}^{\mathrm{n}}$ of dimension $n \leq 3$.

(F) The integrand $F$ satisfies the growth condition $0 \leq F(t) \leq c|t|^{2^{*}}$ for some constant $c$. It is upper semicontinuous and $F \not \equiv 0$ in the $L^{1}$ sense.

$\left(F^{+}\right) \max \left(F_{0}^{+}, F_{\infty}^{+}\right)<S^{F} / S^{*}$ with $F_{0}^{+}, F_{\infty}^{+}$as defined below and $S^{F}, S^{*}$ as in Section 3 . 
The following numbers are used to compare $F$ to the critical power functions.

$$
\begin{aligned}
& F_{0}^{+}:=\limsup _{t \rightarrow 0} \frac{F(t)}{|t|^{2^{*}}}, \quad F_{0}^{-}:=\liminf _{t \rightarrow 0} \frac{F(t)}{|t|^{2^{*}}}, \\
& F_{\infty}^{+}:=\limsup _{|t| \rightarrow \infty} \frac{F(t)}{|t|^{2^{*}}}, \quad F_{\infty}^{-}:=\liminf _{|t| \rightarrow \infty} \frac{F(t)}{|t|^{2^{*}}} .
\end{aligned}
$$

We write $F_{0}:=F_{0}^{+}=F_{0}^{-}$and $F_{\infty}:=F_{\infty}^{+}=F_{\infty}^{-}$in the case of equality.

3. Throughout this paper $\bar{\Omega}$ denotes the closure of $\Omega$ in $\overline{\mathbb{R}^{\mathrm{n}}}=$ $\mathbb{R}^{\mathrm{n}} \cup\{\infty\} \cong S^{n}$. The class of non-negative Borel measures on $\bar{\Omega}$ of finite total mass is denoted by $\mathcal{M}(\bar{\Omega})$. They can be identified with the non-negative elements of $C(\bar{\Omega})^{*}$. Thus convergence in the sense of measures

$$
\mu_{\varepsilon} \stackrel{*}{\rightarrow} \mu \text { in } \mathcal{M}(\bar{\Omega})
$$

is defined by $\int_{\bar{\Omega}} \phi d \mu_{\varepsilon} \rightarrow \int_{\bar{\Omega}} \phi d \mu$ for every test function $\phi \in C(\bar{\Omega})$. To detect the asymptotic behaviour of the extremals $u_{\varepsilon}$ we analyze the limit of the measures

$$
\mu_{\varepsilon}:=\frac{\left|\nabla u_{\varepsilon}\right|^{2} d x}{\int_{\Omega}\left|\nabla u_{\varepsilon}\right|^{2}} \stackrel{*}{\rightarrow} \mu \text { in } \mathcal{M}(\bar{\Omega})
$$

which exists for a subsequence. A priori the limit can be any probability measure on $\bar{\Omega}$. We will show that it is a Dirac measure $\mu=\delta_{x_{0}}$.

\section{GENERALIZED SOBOLEV INEQUALITY}

Definition 1 (Generalized Sobolev constant). - Define

$$
S_{\varepsilon}^{F}(\Omega):=\varepsilon^{-2^{*}} \sup \left\{\int_{\Omega} F(u): u \in D^{1,2}(\Omega),\|\nabla u\|_{2} \leq \varepsilon\right\}
$$

and the generalized Sobolev constant

$$
S^{F}:=S_{1}^{F}\left(\mathbb{R}^{\mathrm{n}}\right) .
$$

Vol. 16, $\pi^{\circ} 3-1999$. 
We say that $\left(u_{\varepsilon}\right)$ is a sequence of almost extremals for $S_{\varepsilon}^{F}(\Omega)$ if $u_{\varepsilon}$ is admissible for the definition of $S_{\varepsilon}^{F}(\Omega)$ and

$$
\varepsilon^{-2^{*}} \int_{\Omega} F\left(u_{\varepsilon}\right)=S_{\varepsilon}^{F}(\Omega)(1+o(1)) \text { as } \varepsilon \rightarrow 0 .
$$

This paper is about almost extremals. They exist by definition of $S_{\varepsilon}^{F}(\Omega)$. We do not discuss the question of existence of exact extremals. The hypothesis $(F)$ implies $0<S^{F} \leq c S^{*}<\infty$. Our extension of the classical Sobolev inequality to general integrands (Lemma 2 below) is a simple consequence of the basic scaling properties of integrals in $\mathbb{R}^{n}$. Define

$$
u^{s}(x):=u(x / s)
$$

Then

$$
\begin{aligned}
& \int_{s \Omega} F\left(u^{s}\right)=s^{n} \int_{\Omega} F(u), \\
& \int_{s \Omega}\left|\nabla u^{s}\right|^{2}=s^{n-2} \int_{\Omega}|\nabla u|^{2} .
\end{aligned}
$$

We will refer to this transformation as it horizontal scaling. In particular it can be used to normalize a function whose Dirichlet integral is not 1 . If we choose $s=\|\nabla u\|_{2}^{-\frac{2}{n-2}}$ then $\left\|\nabla u^{s}\right\|=1$ and

$$
\int_{s \Omega} F\left(u^{s}\right)=\left\|\nabla u^{s}\right\|_{2}^{-2^{*}} \int_{\Omega} F(u) .
$$

Using horizontal scaling it is clear that

$$
S_{s \frac{n-2}{2} \varepsilon}^{F}(s \Omega) \geq S_{\varepsilon}^{F}(\Omega) .
$$

If $\Omega$ is starshaped with respect to the origin then also $S_{s}^{F_{\frac{n-2}{2}}}(\Omega) \geq$ $S_{s^{\frac{n-2}{2}}}^{F}(s \Omega)$ for every $s \leq 1$. This shows that $S_{\varepsilon}^{F^{\prime}}(\Omega)$ is non-increasing in $\varepsilon$ at least for starshaped domains.

LEMma 2 (Generalized Sobolev inequality). - Assume $(\Omega)$ and $(F)$. Then 1. $S_{\varepsilon}^{F}(\Omega) \leq S^{F}$ for every $\varepsilon>0$.

2. In particular the generalized Sobolev inequality

$$
\int_{\Omega} F(u) \leq S^{F}\|\nabla u\|_{2}^{2^{*}}
$$

holds for every domain $\Omega \subset \mathbb{R}^{\mathrm{n}}$ and every $u \in D^{1,2}(\Omega)$. 
3. $S_{\varepsilon}^{F}(\Omega) \rightarrow S^{F}$ as $\varepsilon \rightarrow 0$.

4. $F_{0}^{-} \leq S^{F} / S^{*}, F_{\infty}^{-} \leq S^{F} / S^{*}$.

Proof. - To prove 1. let $u$ be an admissible candidate for the definition of $S_{\varepsilon}^{F}(\Omega)$. The horizontally scaled function $w(x):=u\left(\varepsilon^{\frac{2}{n-2}} x\right)$ is admissible for the definition of $S^{F}$. Therefore

$$
S^{F} \geq \int_{\mathbb{R}^{\mathrm{n}}} F(w)=\varepsilon^{-2^{*}} \int_{\Omega} F(u) .
$$

Taking the supremum over all such $u$ yields 1 . and 2. To prove 3 , fix $\delta>0$, $x_{0} \in \Omega$, and a candidate $w$ for the definition of $S^{F}$ satisfying

$$
\int_{\mathbb{R}^{\mathrm{n}}} F(w) \geq S^{F}-\delta
$$

For $r$ large enough $\int_{B_{0}^{r}} F(w) \geq S^{F}-2 \delta$. In Lemma 8 we construct a cut-off function $\phi_{R}^{r}$ with $\phi_{R}^{r}=1$ in $B_{0}^{r}$ vanishing outside $B_{0}^{R}$ such that $\int_{B_{0}^{R}}\left|\nabla\left(\phi_{R}^{r} w\right)\right|^{2} \leq 1+\delta$. For $\varepsilon$ small enough

$$
u(x):=\left(\phi_{R}^{r} w\right)\left(\left(\frac{1+\delta}{\varepsilon^{2}}\right)^{\frac{1}{n-2}}\left(x-x_{0}\right)\right)
$$

has its support in $\Omega$ and $\|\nabla u\|_{2} \leq \varepsilon$. Thus

$$
\begin{aligned}
S_{\varepsilon}^{F}(\Omega) & \geq \varepsilon^{-2^{*}} \int_{\Omega} F(u) \\
& =(1+\delta)^{-\frac{n}{n-2}} \int_{B_{0}^{R}} F\left(\phi_{R}^{r} w\right) \geq(1+\delta)^{-\frac{n}{n-2}}\left(S^{F}-2 \delta\right)
\end{aligned}
$$

Since $\delta$ was arbitrary we obtain 3 . To prove 4 . choose an extremal $w$ for $S^{*}$. Such extremals exist and are of the form (9) below. The functions $s^{-\frac{n-2}{2}} w^{s}$ are admissible for the definition of $S^{F}$ and they tend uniformly to 0 as $s \rightarrow \infty$. Therefore

$$
\begin{aligned}
S^{F} & \geq \int_{\mathbb{R}^{\mathrm{n}}} F\left(s^{-\frac{n-2}{2}} w^{s}\right) \\
& =s^{n} \int_{\mathbb{R}^{\mathrm{n}}} F\left(s^{-\frac{n-2}{2}} w\right) \geq\left(F_{0}^{-}+o(1)\right) \int_{\mathbb{R}^{\mathrm{n}}}|w|^{2^{*}} .
\end{aligned}
$$

The inequality for $F_{0}^{-}$follows as $s \rightarrow \infty$. Letting $s \rightarrow 0$ we obtain the inequality for $F_{\infty}^{-}$in a similar way. 
For the critical power integrand one has $S_{\varepsilon}^{*}(\Omega)=S^{*}$ for every $\varepsilon$. Typically however, $S_{\varepsilon}^{F}(\Omega)$ decreases as $\varepsilon$ increases. In [5] we show that under suitable assumptions on $F$ including $\left(F^{+}\right)$one has

$$
S_{\varepsilon}^{F}(\Omega)=S^{F}-c^{F} \inf _{\Omega} \tau \varepsilon^{2}+o\left(\varepsilon^{2}\right)
$$

with some constant $c^{F}>0$. Here $\tau$ denotes the value of the Robin function, the regular part of the Green's function with equal arguments [1]. We show that every sequence of low energy extremals concentrates at a minimum point of the Robin function. This is not true for almost extremals. They can concentrate at an arbitrary point in $\bar{\Omega}$.

\section{MAIN RESULTS}

An extremal for the generalized Sobolev constant or entire extremal is a function $w \in D^{1,2}\left(\mathbb{R}^{\mathrm{n}}\right)$ with $\|\nabla w\|_{2}=1$ and $\int_{\mathbb{R}^{\mathrm{n}}} F(w)=S^{F}$. It satisfies an Euler Lagrange equation corresponding to variations of the independent variable. For monotone integrands it is radially symmetric with respect to some point and strictly decreasing or increasing in radial direction. For general integrands this is true outside a compact set. In particular every entire extremal is either strictly positive or strictly negative. These properties together with exact decay rates are derived in [4]. The uniqueness question is unsolved.

THEOREM 3 (Concentration theorem). - Assume $(\Omega)$ and $(F)$ and let $\left(u_{\varepsilon}\right)$ be a sequence of almost extremals for $S_{\varepsilon}^{F}(\Omega)$.

1. If $F_{0}^{+}=F_{0}^{-}$and $\Omega \neq \mathbb{R}^{\mathrm{n}}$ in the sense of capacity, i.e. $\operatorname{cap}_{\mathbb{R}^{\mathrm{n}}}\left(\mathbb{R}^{\mathrm{n}} \backslash \Omega\right)>0$ then a subsequence of $\left(u_{\varepsilon}\right)$ concentrates at some point $x_{0} \in \bar{\Omega}$, i.e.

$$
\begin{gathered}
\frac{\left|\nabla u_{\varepsilon}\right|^{2}}{\varepsilon^{2}} d x \stackrel{*}{\rightarrow} \delta_{x_{0}} \text { in } \mathcal{M}(\bar{\Omega}), \\
\varepsilon^{-2^{*}} F\left(u_{\varepsilon}\right) d x \stackrel{*}{\rightarrow} S^{F} \delta_{x_{0}} \text { in } \mathcal{M}(\bar{\Omega}) .
\end{gathered}
$$

If $F_{0}^{+}<S^{F} / S^{*}$ or if $\Omega$ has finite volume the hypothesis $F_{0}^{+}=F_{0}^{-}$ is not needed.

2. If $\left(F^{+}\right)$holds then there are points $x_{\varepsilon} \rightarrow x_{0}$ such that the rescaled functions

$$
w_{\varepsilon}(y):=u_{\varepsilon}\left(x_{\varepsilon}+\varepsilon^{\frac{2}{n-2}} y\right)
$$


tend to an extremal for $S^{F}$, i.e. $w_{\varepsilon} \rightarrow w$ in $D^{1,2}\left(\mathbb{R}^{\mathrm{n}}\right),\|\nabla w\|_{2}=1$, and $\int_{\mathbb{R}^{\mathrm{n}}} F(w)=1$.

Corollary 4. - Assume $(F)$ and $\left(F^{+}\right)$. Then an extremal for $S^{F}$ exists.

We expect that the conditions $(\Omega),(F)$, and $\Omega \neq \mathbb{R}^{\mathrm{n}}$ already imply concentration. The latter condition is not obsolete. In fact, for the critical power integrand on $\mathbb{R}^{\mathbf{n}}$ the functions

$$
u_{\varepsilon}(x)=\frac{\varepsilon}{\left(c^{2}+|x|^{2}\right)^{\frac{n-2}{2}}}
$$

with suitable $c$ are extremals for $S_{\varepsilon}^{*}\left(\mathbb{R}^{\mathrm{n}}\right)$ but

$$
\frac{\left|\nabla u_{\varepsilon}\right|^{2}}{\varepsilon^{2}} d x=\left|\nabla u_{1}\right|^{2} d x
$$

does not tend to a Dirac measure. Maximizing sequences for the critical power integrand can concentrate arbitrarily fast (Lions [10, Theorem I.1]). This is ruled out by our growth condition at infinity which permits us to prescribe the rate of concentration. Nevertheless the conditions on $\mathrm{F}_{0}^{+}$and $F_{\infty}^{+}$allow for critical growth at 0 and infinity as shown by the following example.

Example 5. - Let

$$
F(t):=G(t)|t|^{2^{*}}, \quad G(t)= \begin{cases}1 & (\mathrm{t} \leq 1 \quad \text { or } \quad \mathrm{t} \geq 4), \\ 2 & (2 \leq \mathrm{t} \leq 3)\end{cases}
$$

and $1 \leq G(t) \leq 2$ else. In this example we have $F_{0}=1, F_{\infty}=1$, and $\left(F^{+}\right)$because $\int_{\mathbb{R}^{n}} F(u)>S^{*}$ for every extremal for $S^{*}$ with $\max u \geq 2$.

\section{APPLICATIONS}

Theorem 3 applies to the following examples.

Example 6 [Volume integrand]. - Set

$$
F(t):= \begin{cases}0 & (\mathrm{t}<1) \\ 1 & (\mathrm{t} \geq 1)\end{cases}
$$

In terms of the set $A:=\{u \leq 1\}$ the variational problem (1) can be written as

$$
\sup \left\{|A|: \operatorname{cap}_{\Omega}(A) \leq \varepsilon^{2}\right\}
$$

Vol. 16, $n^{\circ} 3-1999$. 
because $\int_{\Omega} F(u)=|A|$ and $\int_{\Omega}|\nabla u|^{2}=\operatorname{cap}_{\Omega}(A)$. This form explains the term volume integrand. The weak form of the Euler Lagrange equation is Bernoulli's free-boundary problem: Given the domain $\Omega$ and a number $Q>0$, find a set $A \subset \Omega$ (with the free boundary $\partial A$ ) and a function $u: \Omega \backslash A \rightarrow \mathbb{R}$ such that

$$
\begin{aligned}
-\Delta u & =0 \text { in } \Omega \backslash A, \\
u & =0 \text { on } \partial \Omega, \\
u & =1 \text { on } \partial A, \\
\frac{\partial u}{\partial \nu} & =Q \text { on } \partial A .
\end{aligned}
$$

A derivation of the free-boundary condition together with a detailed analysis of this problem and its applications can be found in [6]. The entire extremals for the volume integrand are given by the translates of

$$
w(x)= \begin{cases}\left(\frac{R}{|x|}\right)^{n-2} & (|x|>\mathrm{R}), \\ 1 & (|x| \leq \mathrm{R})\end{cases}
$$

with $R$ such that $\operatorname{cap}_{\mathbb{R}^{n}}\left(B_{0}^{R}\right)=(n-2)\left|S^{n-1}\right| R^{n-2}=1$. The slope at the free boundary is $Q=(n-2) / R$. This example shows that an entire extremal actually may have flat parts. The generalized Sobolev constant is given by

$$
S^{V}=\int_{\mathbb{R}^{\mathrm{n}}} F(w)=\left|B_{0}^{R}\right|=(n(n-2))^{\frac{n}{2-n}}\left|B_{0}^{1}\right|^{\frac{2}{2-n}} .
$$

In three dimensions $S^{V}=\left(48 \pi^{2}\right)^{-1}$ (Talenti in $[8$, p. 1138]). The generalized Sobolev inequality (5) for the volume integrand is the isoperimetric inequality relating capacity and volume:

$$
|A| \leq S^{V} \operatorname{cap}_{\Omega}^{\frac{n}{n-2}}(A)
$$

In the radial situation $\Omega=B_{0}^{R}$ Bernoulli's free-boundary problem can be solved explicitly. Denote by

$$
K(r)=\frac{1}{(n-2)\left|S^{n-1}\right| r^{n-2}}
$$

the fundamental singularity of the Laplacian. The optimal sets are concentric balls $A_{\varepsilon}=B_{0}^{r}$. The corresponding extremal functions are given by

$$
u_{\varepsilon}(x)=\frac{K(|x|)-K(R)}{K(r)-K(R)} \quad(r \leq|x| \leq R)
$$


extended by 1 in $B_{0}^{r}$ with

$$
\begin{aligned}
\varepsilon^{2} & =\int_{B_{0}^{R}}\left|\nabla u_{\varepsilon}\right|^{2} \\
& =\operatorname{cap}_{B_{0}^{R}}\left(B_{0}^{r}\right)=\frac{1}{K(r)-K(R)}
\end{aligned}
$$

This gives the relation between $\varepsilon$ and $r$. Also on a general domain the optimal sets $A_{\varepsilon}$ concentrate at a single point as follows from Theorem 3 . In [3] $(n=2)$ and [5] $(n \geq 3)$ we show that the concentration point is a minimum point of the Robin function (see also [1, 6]).

Example 7 [Plasma problem]. - In contrast to Bernoulli's free-boundary problem the plasma problem has a continuous integrand vanishing below a certain positive value. Concentration in two dimensions for

$$
F(t)= \begin{cases}(t-1)^{p} & (\mathrm{t} \geq 1) \\ 0 & (\mathrm{t}<1)\end{cases}
$$

with $p \geq 2$ has been shown in [1]. By Theorem 3 concentration also occurs in higher dimensions for every $p \in\left(0,2^{*}\right]$. The corresponding Euler Lagrange equation is

$$
\begin{aligned}
-\Delta u & =\lambda f(u) \text { in } \Omega \backslash \partial A, \\
u & =0 \text { on } \partial \Omega, \\
u & =1 \text { on } \partial A, \\
u & \text { is } C^{1} \text { across } \partial A .
\end{aligned}
$$

More information on this problem for large $\lambda$ can be found in [7]. The entire extremals for the plasma problem in 3 dimensions with $p=2$ are the translates of

$$
w(x)= \begin{cases}\frac{R}{|x|} & (|x|>R) \\ 1+\frac{R}{\pi|x|} \sin \left(\frac{\pi|x|}{R}\right) & (|x| \leq R)\end{cases}
$$

with $R=(6 \pi)^{-1}$.

In both examples we have $F_{0}=0$. Several proofs of this paper can be simplified considerably under this additional hypothesis. 


\section{LOCAL GENERALIZED SOBOLEV INEQUALITY}

Using n-harmonic cut-off functions we derive a local version of the generalized Sobolev inequality. This result will lead to simple and unified proofs of the generalized concentration-compactness alternatives (Theorems 12 and 20) and Theorem 3.

LEMma 8. - For every $\delta>0$ there is a constant $k(\delta)>0$ with the following property. If $0<r<R$ with $r / R \leq k(\delta)$ then there is a cut-off function $\phi_{R}^{r} \in H^{1, \infty}\left(\mathbb{R}^{\mathrm{n}}\right)$ such that $\phi_{R}^{r}=1$ in $B_{x}^{r}$, $\phi_{R}^{r}=0$ outside $B_{x}^{R}$, and

$$
\int_{B_{x}^{R}}\left|\nabla\left(\phi_{R}^{r} u\right)\right|^{2} \leq \int_{B_{x}^{R}}|\nabla u|^{2}+\delta \int_{\mathbb{R}^{\mathrm{n}}}|\nabla u|^{2}
$$

for every $u \in D^{1,2}\left(\mathbb{R}^{\mathrm{n}}\right)$.

Proof. - We may suppose $x=0$. As a cut-off function we choose the $\mathrm{n}$-harmonic capacity potential

$$
\phi_{K}^{r}(x):=\frac{\log (|x| / R)}{\log (r / R)}(r \leq|x| \leq R)
$$

extended by 1 in $B_{0}^{r}$ and hy 0 outside $B_{0}^{R}$. It minimizes the conformal energy

$$
\int_{B_{0}^{R}}\left|\nabla \phi_{R}^{r}\right|^{n}=\frac{|\partial B|}{\left(\log \frac{R}{r}\right)^{n-1}} .
$$

In contrast to the 2-capacity the n-capacity tends to 0 as $R \rightarrow \infty$. By Hölder's and Sobolev's inequality

$$
\begin{aligned}
& \int_{B_{0}^{R}}\left|\nabla\left(\phi_{R}^{r} u\right)\right|^{2} \\
\leq & (1+\beta) \int_{B_{0}^{R}}\left|\phi_{R}^{r}\right|^{2}|\nabla u|^{2}+\left(1+\frac{1}{\beta}\right) \int_{B_{0}^{R}}|u|^{2}\left|\nabla \phi_{R}^{r}\right|^{2} \\
\leq & (1+\beta) \int_{B_{0}^{R}}|\nabla u|^{2}+\left(1+\frac{1}{\beta}\right)\left(\int_{B_{0}^{R}}|u|^{2^{*}}\right)^{\frac{n-2}{n}}\left(\int_{B_{0}^{R}}\left|\nabla \phi_{R}^{r}\right|^{n}\right)^{\frac{2}{n}} \\
\leq & \int_{B_{0}^{R}}|\nabla u|^{2} \\
& +\left[\beta+c_{n}^{2}\left(1+\frac{1}{\beta}\right)\left(\log \frac{R}{r}\right)^{-\frac{2(n-1)}{n}}\right] \int_{\mathbb{R}^{\mathbf{n}}}|\nabla u|^{2}
\end{aligned}
$$


for arbitrary $\beta>0$. With the optimal choice of $\beta$ the square bracket is $\leq \delta$ if

$$
\left(\log \frac{R}{r}\right)^{-\frac{(n-1)}{n}} \leq \frac{\sqrt{1+\delta}-1}{c_{n}}
$$

Hence we can choose

$$
k(\delta):=\exp \left(-\left(\frac{c_{n}}{\sqrt{1+\delta}-1}\right)^{\frac{n}{n-1}}\right) .
$$

CoRollary 9 (Local generalized Sobolev inequality). - Assume $(\Omega)$ and (F). Fix $\delta>0$ and $r / R \leq k(\delta)$ with $k(\delta)$ as in (7). Then

$$
\begin{gathered}
\int_{B_{x}^{r}} F(u) \leq S^{F}\left(\int_{B_{x}^{R}}|\nabla u|^{2}+\delta \int_{\mathbb{R}^{\mathrm{n}}}|\nabla u|^{2}\right)^{\frac{n}{n-2}} \\
\int_{\mathbb{R}^{\mathrm{n}} \backslash B_{x}^{R}} F(u) \leq S^{F}\left(\int_{\mathbb{R}^{n} \backslash B_{x}^{r}}|\nabla u|^{2}+\delta \int_{\mathbb{R}^{\mathrm{n}}}|\nabla u|^{2}\right)^{\frac{n}{n-2}} .
\end{gathered}
$$

for every $u \in D^{1,2}\left(\mathbb{R}^{\mathrm{n}}\right)$.

Proof. - We may suppose $x=0$. By Lemma 8 and the generalized Sobolev inequality (5) (note that $\phi_{R}^{r} u$ vanishes on $\partial B_{0}^{R}$ ) we have

$$
\begin{aligned}
\int_{B_{0}^{r}} F(u) & \leq \int_{B_{0}^{R}} F\left(\phi_{R}^{r} u\right) \leq S^{F}\left\|\nabla\left(\phi_{R}^{r} u\right)\right\|_{2}^{2^{*}} \\
& \leq S^{F}\left(\int_{B_{0}^{R}}|\nabla u|^{2}+\delta \int_{\mathbb{R}^{\mathrm{n}}}|\nabla u|^{2}\right)^{\frac{n}{n-2}}
\end{aligned}
$$

For the second inequality we use the cut-off function $\left(1-\phi_{R}^{r}\right) \phi_{R_{2}}^{R_{1}}$ with $r<R<R_{1}<R_{2}$ and let $R_{1}$ and $R_{2} \rightarrow \infty$ such that $R_{2} / R_{1} \rightarrow \infty$.

\section{GENERALIZED CONCENTRATION- COMPACTNESS ALTERNATIVES}

The critical power integrand has a special invariance. Namely $u$ and $x \mapsto s^{-(n-2) / 2} u(x / s)$ have the same Dirichlet integral and $L^{2^{*}}$ norm. For this reason the critical Sobolev embedding is noncompact on bounded Vol, 16, n 3-1999. 
domains. The failure of compactness can be described precisely. Every maximizing sequence concentrates at one point. This follows from a concentration-compactness alternative of P.L. Lions (Lemma 10 below). We quote it in a form adapted to our purpose. In particular we allow for concentration at infinity.

LEMma 10 (P. L. Lions $[10$, p. 158]). - Assume $(\Omega)$ and consider a sequence $\left(v_{\varepsilon}\right)$ in $D^{1,2}(\Omega)$ with

$$
\begin{aligned}
\left\|\nabla v_{\varepsilon}\right\|_{2} & \leq 1 \\
v_{\varepsilon} & -v_{0} \text { weakly in } D^{1,2}(\Omega), \\
\left|\nabla v_{\varepsilon}\right|^{2} d x & \stackrel{*}{\rightarrow} \mu \text { in } \mathcal{M}(\bar{\Omega}) \\
\left|v_{\varepsilon}\right|^{2^{*}} d x & \stackrel{*}{\rightarrow} \nu^{*} \text { in } \mathcal{M}(\bar{\Omega})
\end{aligned}
$$

Then:

1. The limit measures are of the form

$$
\begin{aligned}
\mu & =\left|\nabla v_{0}\right|^{2} d x+\sum_{j=1}^{J} \mu_{j} \delta_{x_{j}}+\tilde{\mu}, \quad \mu(\bar{\Omega}) \leq 1, \\
\nu^{*} & =\left|v_{0}\right|^{2^{*}} d x+\sum_{j=1}^{J} \nu_{j}^{*} \delta_{x_{j}}, \quad \nu(\bar{\Omega}) \leq S^{*}
\end{aligned}
$$

with $J \in \mathbb{N} \cup\{\infty\}$ and a nonatomic $\tilde{\mu} \in \mathcal{M}(\bar{\Omega})$. Moreover

$$
\left|v_{\varepsilon}-v_{0}\right|^{2^{*}} d x \stackrel{*}{\rightarrow} \sum_{j=1}^{J} \nu_{j}^{*} \delta_{x_{j}} \text { in } \mathcal{M}(\bar{\Omega})
$$

The atoms satisfy the Sobolev inequality

$$
0 \leq \nu_{j}^{*} \leq S^{*} \mu_{j}^{\frac{n}{n-2}}
$$

2. If $\nu^{*}(\bar{\Omega})=S^{*}$ then $\mu(\bar{\Omega})=1$ and one of the following statements holds true.

(a) Concentration: $\mu$ and $\nu^{*}$ are concentrated at a single point, i.e. $v_{0}=0, \mu=\delta_{x_{0}}$, and $\nu=S^{*} \delta_{x_{0}}$ for some $x_{0} \in \bar{\Omega}$.

(b) Compactness: $v_{\varepsilon} \rightarrow v_{0}$ in $D^{1,2}(\Omega), \mu=\left|\nabla v_{0}\right|^{2} d x$, and $\nu^{*}=$ $\left|v_{0}\right|^{2^{*}} d x$. 
The key point in the proof of 2 . is the following convexity argument. With

one has

$$
\mu_{0}:=\int_{\Omega}\left|\nabla v_{0}\right|^{2}, \quad \nu_{0}^{*}:=\int_{\Omega}\left|v_{0}\right|^{2^{*}}
$$

$$
\begin{aligned}
\left(\sum_{j=0}^{J} \mu_{j}\right)^{\frac{n}{n-2}} & \leq(\mu(\bar{\Omega}))^{\frac{n}{n-2}} \\
& =\frac{1}{S^{*}} \nu^{*}(\bar{\Omega})=\frac{1}{S^{*}} \sum_{j=0}^{J} \nu_{j}^{*} \leq \sum_{j=0}^{J} \mu_{j}^{\frac{n}{n-2}}
\end{aligned}
$$

by Sobolev's inequality (3) for the regular part and for the atoms (8). By strict convexity of the function $t \mapsto t^{\frac{n}{n-2}}$ on $\mathbb{R}^{+}$only one of the $\mu_{j}$ 's can be nonzero. Hence either concentration or compactness occurs. Lemma 10 applies to maximizing sequences for the critical power integrand.

Example 11 [Critical power integrand]. - Assume $\Omega \neq \mathbb{R}^{\mathrm{n}}$ in the sense of capacity, i.e. $\operatorname{cap}_{\mathbb{I R}^{\mathrm{n}}}\left(\mathbb{R}^{\mathrm{n}} \backslash \Omega\right)>0$ and suppose $\left\|\nabla v_{\varepsilon}\right\|_{2} \leq 1, \int_{\Omega}\left|v_{\varepsilon}\right|^{2^{*}} \rightarrow S^{*}$. Then $\nu^{*}(\bar{\Omega})=S^{*}$. If alternative (b) would hold then the optimal Sobolev constant would be attained by a function vanishing on $\mathbb{R}^{n} \backslash \Omega$. This is impossible because all extremals for $S^{*}$ are of the form

$$
v(x)=\frac{c}{\left(s+\frac{\left|x-x_{0}\right|^{2}}{s}\right)^{\frac{n-2}{2}}}
$$

with $x_{0} \in \mathbb{R}^{\mathrm{n} 1}, s>0$, and a constant $c$ (see Talenti [12]). In three dimensions $c=2 /(\sqrt{3} \pi)$. It follows that alternative (a) must hold, i.e. that a subsequence of $\left(v_{\varepsilon}\right)$ concentrates at a single point $x_{0} \in \bar{\Omega}, \mu=\delta_{x_{0}}$, and $\nu^{*}=S^{*} \delta_{x_{0}}$. On the other hand it is possible to construct a maximizing sequence for $S^{*}$ concentrating at an arbitrary prescribed point $x_{0} \in \bar{\Omega}$.

Our aim is to extend this conclusion (concentration of maximizing sequences) to general nonlinearities. The following generalized concentration-compactness alternative is the basis of part 1 of Theorem 3 .

THEOREM 12 (Generalized concentration-compactness alternative I). Assume $(\Omega)$ and $(F)$. Let $\left(u_{\varepsilon}\right)$ be a sequence in $D^{1,2}(\Omega)$ with $\left\|\nabla u_{\varepsilon}\right\|_{2} \leq \varepsilon$ and define $v_{\varepsilon}:=u_{\varepsilon} / \varepsilon$. Assume

$$
\begin{aligned}
v_{\varepsilon} & \rightarrow v_{0} \text { weakly in } D^{1,2}(\Omega), \\
\left|\nabla v_{\varepsilon}\right|^{2} d x & \stackrel{*}{-} \mu \text { in } \mathcal{M}(\bar{\Omega}), \\
\varepsilon^{-2^{*}} F\left(u_{\varepsilon}\right) d x & \stackrel{*}{-} \nu \text { in } \mathcal{M}(\bar{\Omega}) .
\end{aligned}
$$

Vol. 16, n $n^{\circ}$ 3-1999. 
Then:

1. The limit measures are of the form

$$
\begin{aligned}
& \mu=\left|\nabla v_{0}\right|^{2} d x+\sum_{j=1}^{J} \mu_{j} \delta_{x_{j}}+\tilde{\mu}, \quad \mu(\bar{\Omega}) \leq 1 \\
& \nu=g d x+\sum_{j=1}^{J} \nu_{j} \delta_{x_{j}}, \quad \nu(\bar{\Omega}) \leq S^{F}
\end{aligned}
$$

with $J \in \mathbb{N} \cup\{\infty\}$, a nonatomic $\tilde{\mu} \in \mathcal{M}(\bar{\Omega})$, and $g \in L^{1}(\Omega)$. The total mass, the atoms, and the regular part satisfy the generalized Sobolev inequality

$$
\begin{aligned}
\nu(\bar{\Omega}) & \leq S^{F}(\mu(\bar{\Omega}))^{\frac{n}{n-2}} \\
\nu_{j} & \leq S^{F} \mu_{j}^{\frac{n}{n-2}} \\
\int_{\Omega} g & \leq S^{F}\left(\int_{\Omega}\left|\nabla v_{0}\right|^{2}+\tilde{\mu}(\bar{\Omega})\right)^{\frac{n}{n-2}} \\
g & \leq F_{0}^{+}\left|v_{0}\right|^{2^{*}} \text { a.e. in } \Omega \\
\int_{\Omega} g & \leq F_{0}^{+} S^{*}\left(\int_{\Omega}\left|\nabla v_{0}\right|^{2}\right)^{\frac{n}{n-2}} .
\end{aligned}
$$

2. If $\nu(\bar{\Omega})=S^{F}$ then $\mu(\bar{\Omega})=1$ and one of the following statements holds true.

(a) Concentration: $v_{0}=0, \mu=\delta_{x_{0}}$, and $\nu=S^{F} \delta_{x_{0}}$ for some $x_{0} \in \bar{\Omega}$.

(b) Compactness:

$$
\begin{aligned}
\mu & =\left|\nabla v_{0}\right|^{2} d x+\tilde{\mu}, \\
v_{\varepsilon} & \rightarrow v_{0} \text { in } L^{2^{*}}(\Omega), \quad S^{F} \leq c \int_{\Omega}\left|v_{0}\right|^{2^{*}}, \\
\varepsilon^{-2^{*}} F\left(u_{\varepsilon}\right) & \rightarrow g \text { weakly in } L^{1}(\Omega), \\
S^{F} & =\int_{\Omega} g \leq F_{0}^{+} S^{*} .
\end{aligned}
$$

If in addition $F_{0}^{+}=F_{0}^{-}$then $v_{\varepsilon} \rightarrow v_{0}$ in $D^{1,2}(\Omega), S^{F}=F_{0} S^{*}$, $v_{0}$ is an extremal for $S^{*}$ and $\Omega=\mathbb{R}^{n}$ up to a set of capacity zero.

Proof. - The proof of Theorem 12 is divided into a number of steps. 
STEP 13 (Generalized Sobolev inequality for total mass). - The total mass of $\mu$ and $\nu$ satisfy

$$
\nu(\bar{\Omega}) \leq S^{F}(\mu(\bar{\Omega}))^{\frac{n}{n-2}}
$$

Proof. - By the generalized Sobolev inequality (5)

$$
\int_{\Omega} \varepsilon^{-2^{*}} F\left(u_{\varepsilon}\right) \leq S^{F}\left(\int_{\Omega}\left|\nabla v_{\varepsilon}\right|^{2}\right)^{\frac{n}{n-2}} .
$$

Passing to the limit $\varepsilon \rightarrow 0$ yields the claim since $\phi \equiv 1$ is an admissible test function for convergence in $\mathcal{M}(\bar{\Omega})$.

STEP 14 (Decomposition of $\mu$ and $\nu$ ). - The limit measures $\mu$ and $\nu$ are of the form

$$
\begin{aligned}
& \mu=\left|\nabla v_{0}\right|^{2} d x+\sum_{j=1}^{J} \mu_{j} \delta_{x_{j}}+\tilde{\mu}, \\
& \nu=g d x+\sum_{j=1}^{J} \nu_{j} \delta_{x_{j}}
\end{aligned}
$$

with $J \in \mathbb{N} \cup\{\infty\}$, a nonatomic $\tilde{\mu} \in \mathcal{M}(\bar{\Omega})$, and $g \in L^{1}(\Omega)$.

Proof. - For a subsequence we have

$$
\left|v_{\varepsilon}\right|^{2^{*}} d x \stackrel{*}{\rightarrow} \nu^{*} \text { in } \mathcal{M}(\bar{\Omega}) .
$$

From Lions' result for the critical power integrand (Lemma 10) we know that $\mu$ is of the above form and

$$
\begin{array}{r}
\nu^{*}=\left|v_{0}\right|^{2^{*}} d x+\sum_{j=1}^{J} \nu_{j}^{*} \delta_{x_{j}}, \\
\left|v_{\varepsilon}-v_{0}\right|^{2^{*}} d x \stackrel{*}{\rightarrow} \sum_{j=1}^{J} \nu_{j}^{*} \delta_{x_{j}} \text { in } \mathcal{M}(\bar{\Omega}) .
\end{array}
$$

By our growth condition $(F)$ we have $0 \leq \nu \leq c \nu^{*}$. Application of the Radon Nikodym theorem with respect to the measure $\nu^{*}$ yields

$$
\nu=g d x+\sum_{j=1}^{J} \nu_{j} \delta_{x_{j}}
$$

with $g \in L^{1}(\Omega)$.

Vol. 16, $\mathrm{n}^{\circ}$ 3-1999. 
STEP 15 (Generalized Sobolev inequality for atoms). - For every $j \geq 1$

$$
\nu_{j} \leq S^{F} \mu_{j}^{\frac{n}{n-2}} .
$$

Proof. - Let $\delta>0$ and $R>0$. If $x_{j} \in \mathbb{R}^{n}$ it follows from the local generalized Sobolev inequality that

$$
\nu_{j} \leq \nu\left(\overline{B_{x_{j}}^{r}}\right) \leq S^{F}\left(\mu\left(\overline{B_{x_{j}}^{R}}\right)+\delta\right)^{\frac{n}{n-2}}
$$

for $r \leq k(\delta) R$. The assertion follows as $r \rightarrow 0, \delta \rightarrow 0$, and $R \rightarrow 0$. If $x_{j}=\infty$ we apply the second inequality of Corollary 9 .

STEP 16 (Generalized Sobolev inequality for regular part). - The regular part $g$ of $\nu$ satisfies

$$
\int_{\Omega} g \leq S^{F}\left(\int_{\Omega}\left|\nabla v_{0}\right|^{2}+\tilde{\mu}(\bar{\Omega})\right)^{\frac{n}{n-2}} .
$$

Proof. - This step is complementary to the previous one. Now we excise the atoms. Fix $\delta>0, R_{1}>0$, and a finite $I \leq J$ such that $\sum_{j=I+1}^{J} \mu_{j} \leq \delta$. Choose $R>0$ so small that the balls $B_{x_{1}}^{R}, \ldots, B_{x_{I}}^{R}$ are disjoint. The cut-off function

$$
\phi:=\phi_{R_{2}}^{R_{1}} \prod_{i=1}^{I}\left(1-\phi_{R}^{r}\left(\cdot-x_{i}\right)\right)
$$

is supported in $B_{0}^{R_{2}} \backslash \bigcup_{i=1}^{I} B_{x_{i}}^{r}$. By the local generalized Sobolev inequality there exist $r>0$ and $R_{2}>R_{1}$ such that

$$
\begin{aligned}
& \int_{B_{0}^{R_{1}} \backslash \bigcup_{i=1}^{I} B_{x_{i}}^{R}} g \\
\leq & \nu\left(B_{0}^{R_{1}} \backslash \bigcup_{i=1}^{I} B_{x_{i}}^{R}\right) \leq S^{F}\left(\mu\left(B_{0}^{R_{2}} \backslash \bigcup_{i=1}^{I} B_{x_{i}}^{r}\right)+\delta\right)^{\frac{n}{n-2}} \\
\leq & S^{F}\left(\int_{\Omega}\left|\nabla v_{0}\right|^{2}+\tilde{\mu}(\bar{\Omega})+\delta\right)^{\frac{n}{n-2}} .
\end{aligned}
$$

Then let $R \rightarrow 0, R_{1} \rightarrow \infty$, and $\delta \rightarrow 0$.

We even have the following pointwise estimate.

STEP 17 (Pointwise estimate of regular part)

$$
\begin{aligned}
g & \leq F_{0}^{+}\left|v_{0}\right|^{2^{*}} \text { a.e. in } \Omega, \\
\int_{\Omega} g & \leq F_{0}^{+} S^{*}\left(\int_{\Omega}\left|\nabla v_{0}\right|^{2}\right)^{\frac{n}{n-2}} .
\end{aligned}
$$


If $F_{0}^{+}=F_{0}^{-}$then

$$
\int_{\Omega} g \leq S^{F}\left(\int_{\Omega}\left|\nabla v_{0}\right|^{2}\right)^{\frac{n}{n-2}} .
$$

Proof. - The pointwise estimate of $g$ is based on the fact that the large values of the $v_{\varepsilon}$ do not contribute to the regular part. Let $U \subset \mathbb{R}^{\mathrm{n}}$ be an open set. For $t>0$ we have

$$
\begin{aligned}
\int_{U} g \leq & \nu(U) \\
\leq & \liminf _{\varepsilon \rightarrow 0} \int_{U} \varepsilon^{-2^{*}} F\left(u_{\varepsilon}\right) \\
\leq & \limsup _{\varepsilon \rightarrow 0} \int_{U \cap\left\{\left|v_{\varepsilon}\right|<t\right\}} \varepsilon^{-2^{*}} F\left(u_{\varepsilon}\right)+c \limsup _{\varepsilon \rightarrow 0} \int_{U \cap\left\{\left|v_{\varepsilon}\right| \geq t\right\}}\left|v_{\varepsilon}\right|^{2^{*}} \\
\leq & \left(F_{0}^{+}+o(1)\right) \nu^{*}(\bar{U}) \\
& \quad+c 2^{2^{*}} \limsup _{\varepsilon \rightarrow 0}\left(\int_{U \cap\left\{\left|v_{\varepsilon}\right| \geq t\right\}}\left|v_{0}\right|^{2^{*}}+\int_{U}\left|v_{\varepsilon}-v_{0}\right|^{2^{*}}\right) .
\end{aligned}
$$

Since

$$
\lim _{t \rightarrow \infty} \sup _{\varepsilon>0}\left|\left\{\left|v_{\varepsilon}\right| \geq t\right\}\right|=0
$$

we deduce in combination with Lemma 10 that

$$
\begin{aligned}
\int_{U} g & \leq F_{0}^{+} \nu^{*}(\bar{U})+2^{2^{*}} c \sum_{x_{j} \in \bar{U}} \nu_{j}^{*} \\
& \leq F_{0}^{+} \int_{U}\left|v_{0}\right|^{2^{*}}+\left(1+2^{2^{*}} c\right) \sum_{x_{j} \in \bar{U}} \nu_{j}^{*} .
\end{aligned}
$$

Application of the Radon-Nikodym theorem yields $g \leq F_{0}^{+}\left|v_{0}\right|^{2^{*}}$ a.e. The second inequality follows by integration and the standard Sobolev inequality. The last inequality follows from the second one and the inequality $F_{0}^{-} S^{*} \leq S^{F}$.

The proof of part 1 of Theorem 12 is complete.

STEP 18 (Part 2 of Theorem 12).

Proof. - Let $\nu_{0}:=\int_{\Omega} g$ and $\mu_{0}:=\int_{\Omega}\left|\nabla v_{0}\right|^{2}+\tilde{\mu}(\bar{\Omega})$. By Step 13 and normalization of $v_{\varepsilon}$ we deduce

$$
S^{F}=\nu(\bar{\Omega}) \leq S^{F}(\mu(\bar{\Omega}))^{\frac{n}{n-2}} \leq S^{F} .
$$

Vol. $16, n^{\circ}$ 3-1999. 
Hence $\mu(\bar{\Omega})=1$. By the Steps 13,15 and 16

$$
\left(\sum_{j=0}^{J} \mu_{j}\right)^{\frac{n}{n-2}}=(\mu(\bar{\Omega}))^{\frac{n}{n-2}}=\frac{\nu(\bar{\Omega})}{S^{F}} \leq \sum_{j=0}^{J} \mu_{j}^{\frac{n}{n-2}}
$$

Strict convexity of $t \mapsto t^{\frac{n}{n-2}}$ on $\mathbb{R}^{+}$implies that all but one of the $\mu_{j}$ vanish. If $\mu_{0}=0$ then alternative (a) holds and we are done. If $\mu_{0} \neq 0$, then $\nu_{j}^{*}=\nu_{j}=\mu_{j}=0$ for every $j \geq 1, \mu_{0}=1, \int_{\Omega} g=\nu_{0}=S^{F}$. From Lemma 10 we know that $\nu^{*}=\left|v_{0}\right|^{2^{*}} d x$ and $\int_{U}\left|v_{\varepsilon}\right|^{2^{*}} \rightarrow \int_{U}\left|v_{0}\right|^{2^{*}}$ for every bounded measurable set $U$. Together with weak convergence we get $v_{\varepsilon} \rightarrow v_{0}$ in $L_{\mathrm{loc}}^{2^{*}}(\Omega)$. The measure $\mu$ is nonatomic. In particular $\mu(\{\infty\})=0$. Hence $\nu^{*}(\{\infty\})=0$ and

$$
v_{\varepsilon} \rightarrow v_{0} \text { in } L^{2^{*}}(\Omega)
$$

By our growth condition $F\left(u_{\varepsilon}\right) / \varepsilon^{2^{*}} \leq c\left|v_{\varepsilon}\right|^{2^{*}} \rightarrow c\left|v_{0}\right|^{2^{*}}$ in $L^{1}(\Omega)$. Thus the functions $\left(F\left(u_{\varepsilon}\right) / \varepsilon^{2^{*}}\right)$ are equi-integrable. By the Dunford-Pettis compactness criterion (see e.g. Dellacherie and Meyer [2, Theorem 25])

$$
\varepsilon^{-2^{*}} F\left(u_{\varepsilon}\right) \rightarrow g \text { weakly in } L^{1}(\Omega)
$$

for a subsequence. Although we know that $\int_{\Omega} g=S^{F}=\lim \int_{\Omega} F\left(u_{\varepsilon}\right) / \varepsilon^{2^{+}}$ we do not obtain $L^{1}$ convergence because the $L^{1}$ norm is not strictly convex.

Now assume $F_{0}^{+}=F_{0}^{-}=F_{0}$. We may assume $F_{0}>0$ since otherwise $S^{F}=0$ which contradicts the hypothesis $F \not \equiv 0$. By Step 17

$$
S^{F}=\nu_{0} \leq S^{F}\left(\int_{\Omega}\left|\nabla v_{0}\right|^{2}\right)^{\frac{n}{n-2}} .
$$

Hence $\left\|\nabla v_{0}\right\|_{2}=1$. Since $\nabla v_{\varepsilon} \rightarrow \nabla v_{0}$ weakly in $L^{2}(\Omega)$ it follows that $v_{\varepsilon} \rightarrow v_{0}$ strongly in $D^{1,2}(\Omega)$. We show that $\left(v_{\varepsilon}\right)$ is a maximizing sequence for $S^{*}$. Fix $t>0$. We estimate

$$
\begin{aligned}
\int_{\Omega} \varepsilon^{-2^{+}} F^{\prime}\left(u_{\varepsilon}\right) & \leq \int_{\left\{\left|v_{\varepsilon}\right| \leq t\right\}} \varepsilon^{-2^{*}} F^{\prime}\left(\varepsilon v_{\varepsilon}\right)+c \int_{\left\{\left|v_{\varepsilon}\right| \geq t\right\}}\left|v_{\varepsilon}\right|^{2^{+}} \\
& \leq\left(F_{0}^{+}+o(1)\right) \int_{\Omega}\left|v_{\varepsilon}\right|^{2^{+}}+c \int_{\Omega} h_{t}\left(v_{\varepsilon}\right) \text { as } \cdot \varepsilon \rightarrow 0
\end{aligned}
$$

with

$$
h_{t}(s):= \begin{cases}|s|^{2^{*}} & (|s| \geq t) \\ 0 & \text { (otherwise) }\end{cases}
$$


By Fatou's lemma applied to the functions $\left|v_{\varepsilon}\right|^{2^{*}}-h_{t}\left(v_{\varepsilon}\right) \geq 0$, upper semi continuity of $h_{t}$, and the fact that $v_{\varepsilon} \rightarrow v_{0}$ a.e. the second term is asymptotically dominated by

$$
\begin{aligned}
\limsup _{\varepsilon \rightarrow 0} \int_{\Omega} h_{t}\left(v_{\varepsilon}\right) & \leq \int_{\Omega} \limsup _{\varepsilon \rightarrow 0} h_{t}\left(v_{\varepsilon}\right) \\
& \leq \int_{\Omega} h_{t}\left(v_{0}\right)=\int_{\left\{\left|v_{0}\right| \geq t\right\}}\left|v_{0}\right|^{2^{*}} .
\end{aligned}
$$

Letting first $\varepsilon \rightarrow 0$ and then $t \rightarrow \infty$ we obtain

$$
\begin{aligned}
F_{0}^{-} S^{*} & \leq S^{F}=\lim _{\varepsilon \rightarrow 0} \int_{\Omega} \varepsilon^{-2^{*}} F\left(u_{\varepsilon}\right) \\
& \leq F_{0}^{+} \lim _{\varepsilon \rightarrow 0} \int_{\Omega}\left|v_{\varepsilon}\right|^{2^{*}} \leq F_{0}^{+} S^{*}
\end{aligned}
$$

by Sobolev's inequality and normalization of $v_{\varepsilon}$. Since $F_{0}^{-}=F_{0}^{+}>0$ the sequence $\left(v_{\varepsilon}\right)$ is maximizing for $S^{*}$. The assertion follows from the hypothesis $\operatorname{cap}_{\mathbb{R}^{\mathrm{n}}}\left(\mathbb{R}^{\mathrm{n}} \backslash \Omega\right)>0$ and Example 11 .

The proof of the generalized concentration-compactness alternative I is complete.

In particular we found that $\nu(\bar{\Omega})=S^{F}>F_{0}^{+} S^{*}$ implies concentration. For power nonlinearities compactness holds if and only if $F_{0}=\infty$. For general integrands this condition is not enough to rule out concentration as shown by the following example.

Example 19. - Consider

$$
F(t)=\log \left(\frac{1}{|t|}+e\right)|t|^{2^{*}}
$$

with $F_{\infty}=1, F_{0}=\infty$ on a domain $\Omega \neq \mathbb{R}^{\mathrm{n}}$ in the sense of capacity. We claim that every sequence of almost extremals $\left(u_{\varepsilon}\right)$ for $S_{\varepsilon}^{F}(\Omega)$ forms a maximizing sequence for the critical power integrand. Thus they concentrate hy Example 11. To see this we rescale $v_{\varepsilon}=u_{\varepsilon} / \varepsilon$. Then

$$
S_{\varepsilon}^{F}(\Omega)-o(1)=\varepsilon^{-2^{*}} \int_{\Omega} F\left(\varepsilon v_{\varepsilon}\right)=-\log (\varepsilon) \int_{\Omega} g_{\varepsilon}\left(v_{\varepsilon}\right)
$$

where

$$
g_{\varepsilon}(t)=|t|^{2^{*}}-\frac{\log \left(|t|^{-1}+\varepsilon e\right)}{\log (\varepsilon)}|t|^{2^{*}} .
$$


Since $\left(u_{\varepsilon}\right)$ is a sequence of almost extremals for $S_{\varepsilon}^{F}(\Omega)$, so is $\left(v_{\varepsilon}\right)$ for $S_{1}^{g_{\varepsilon}}(\Omega)$. A simple estimate yields

$$
g_{\varepsilon}(t)=|t|^{2^{*}}(1+o(1))+o(1) \text { as } \varepsilon \rightarrow 0
$$

uniformly in $t$. Choose an arbitrary maximizing sequence $\left(\bar{v}_{\varepsilon}\right)$ for $S^{*}$. By almost optimality of $v_{\varepsilon}$ we have

$$
\begin{aligned}
\int_{\Omega}\left|v_{\varepsilon}\right|^{2^{*}} & =(1+o(1)) \int_{\Omega} g_{\varepsilon}\left(v_{\varepsilon}\right)+o(1) \\
& \geq(1+o(1)) \int_{\Omega} g_{\varepsilon}\left(\bar{v}_{\varepsilon}\right)+o(1) \\
& \geq(1+o(1)) \int_{\Omega}\left|\bar{v}_{\varepsilon}\right|^{2^{*}}+o(1)=S^{*}+o(1) .
\end{aligned}
$$

This shows that $\left(v_{\varepsilon}\right)$ is a sequence of almost extremals for $S^{*}$. Thus it concentrates.

The following variant of the generalized concentration-compactness alternative $I$ is used to analyze the asymptotic behaviour of almost extremals near the concentration point.

THEOREM 20 (Generalized concentration-compactness alternative II). Assume $(\Omega)$ and $(F)$. Let $\left(w_{\varepsilon}\right)$ be a sequence in $D^{1,2}(\Omega)$ of norm $\left\|\nabla w_{e}\right\|_{2} \leq 1$ such that

$$
\begin{aligned}
& w_{\varepsilon} \rightarrow w \text { weakly in } D^{1,2}(\Omega), \\
&\left|\nabla w_{\varepsilon}\right|^{2} d y \stackrel{*}{\rightarrow} \mu \text { in } \mathcal{M}(\bar{\Omega}) \\
& F\left(w_{\varepsilon}\right) d y \stackrel{*}{\rightarrow} \nu \text { in } \mathcal{M}(\bar{\Omega})
\end{aligned}
$$

Then:

1. The limit measures are of the form

$$
\begin{aligned}
& \mu=|\nabla w|^{2} d x+\sum_{j=1}^{J} \mu_{j} \delta_{x_{j}}+\tilde{\mu}, \quad \mu(\bar{\Omega}) \leq 1 \\
& \nu=g d x+\sum_{j=1}^{J} \nu_{j} \delta_{x_{j}}, \quad \nu(\bar{\Omega}) \leq S^{F}
\end{aligned}
$$

with $J \in \mathbb{N} \cup\{\infty\}$, a nonatomic $\tilde{\mu} \in \mathcal{M}(\bar{\Omega})$, and $g \in L^{1}(\Omega)$ such that

$$
\begin{aligned}
\nu(\bar{\Omega}) & \leq S^{F}(\mu(\bar{\Omega}))^{\frac{n}{n-2}}, \\
\nu_{j} & \leq S^{F} \mu_{j}^{\frac{n}{n-2}} \\
\int_{\Omega} g & \leq S^{F}\left(\int_{\Omega}|\nabla w|^{2}+\tilde{\mu}(\bar{\Omega})\right)^{\frac{n}{n-2}} .
\end{aligned}
$$


2. If $\nu(\bar{\Omega})=S^{F}$ then $\mu(\bar{\Omega})=1$ and one of the following statements holds true.

(a) Concentration: $w=0, \mu=\delta_{x_{0}}$, and $\nu=S^{F} \delta_{x_{0}}$ for some $x_{0} \in \bar{\Omega}$.

(b) Compactness: $w_{\varepsilon} \rightarrow w$ in $D^{1,2}(\Omega), w$ is an extremal for $S^{F}$, $\mu=|\nabla w|^{2} d x$, and $F\left(w_{\varepsilon}\right) \rightarrow F(w)$ in $L^{1}(\Omega)$.

Compactness can only occur if $\Omega=\mathbb{R}^{\mathrm{n}}$ up to a set of capacity zero.

Proof. - The proof of Theorem 20 is similar to that of the first alternative with the following exceptions. Step 17 is omitted and the following ones are added.

STEP 21. - In the compactness case we have strong convergence $w_{\varepsilon} \rightarrow w$ in $D^{1,2}(\Omega), w$ is an extremal for $S^{F}$, and $F\left(w_{\varepsilon}\right) \rightarrow F(w)$ in $L^{1}(\Omega)$.

Proof. - For a subsequence $w_{\varepsilon} \rightarrow w$ a.e. By upper semicontinuity of $F$ and application of Fatou's lemma to the sequence $\left(c\left|w_{\varepsilon}\right|^{2^{*}}-F\left(w_{\varepsilon}\right)\right)$ it follows that

$$
\begin{aligned}
S^{F} & =\lim _{\varepsilon \rightarrow 0} \int_{\Omega} F\left(w_{\varepsilon}\right) \\
& \leq \int_{\Omega} \limsup _{\varepsilon \rightarrow 0} F\left(w_{\varepsilon}\right) \leq \int_{\Omega} F(w) \leq S^{F}\|\nabla w\|_{2}^{2^{*}} \leq S^{F} .
\end{aligned}
$$

Hence $\|\nabla w\|_{2}=1$ which implies strong convergence. Moreover $w$ is an entire extremal. Equality in the above chain of inequalities implies

$$
\limsup _{\varepsilon \rightarrow 0} F\left(w_{\varepsilon}\right)=F(w) \text { a.e. }
$$

By the same arguments as in Step 18 we have

$$
F\left(w_{\varepsilon}\right) \rightarrow g \text { in } L^{1}(\Omega)
$$

for a subsequence. Moreover $g \leq F(w)$ a.e. by upper semicontinuity of $F$. On the other hand $\int_{\Omega} F(w)=S^{F}=\int_{\Omega} g$ and thus $g=F(w)$ a.e. By (10) we have $\left(F\left(w_{\varepsilon}\right)-F(w)\right)^{+} \rightarrow 0$ a.e. Since $0 \leq F\left(w_{\varepsilon}\right) \leq c\left|w_{\varepsilon}\right|^{2^{*}} \rightarrow c|w|^{2^{*}}$ in $L^{1}(\Omega)$ a suitable version of Lebesgue's dominated convergence theorem implies $\left(F\left(w_{\varepsilon}\right)-F(w)\right)^{+} \rightarrow 0$ in $L^{1}(\Omega)$. By (11) the same is true for the negative part:

$$
\int_{\Omega}\left(F\left(w_{\varepsilon}\right)-F(w)\right)^{-}=\int_{\Omega}\left(F\left(w_{\varepsilon}\right)-F(w)\right)-\int_{\Omega}\left(F\left(w_{\varepsilon}\right)-F(w)\right)^{+}
$$

which tends to 0 . This completes the proof.

STEP 22. - Compactness can only occur if $\Omega=\mathbb{R}^{\mathrm{n}}$ up to a set of capacity zero.

Proof. - This follows from the fact that every entire extremal is strictly positive or strictly negative in all of $\mathbb{R}^{\mathrm{n}}[4]$. 


\section{PROOF OF THE CONCENTRATION THEOREM}

\subsection{Concentration}

We show that Theorem 12 implies part 1 of Theorem 3. Let $\left(u_{\varepsilon}\right)$ be a sequence of almost extremals for $S_{\varepsilon}^{F}(\Omega)$. Then a subsequence of $\left(u_{\varepsilon}\right)$ satisfies the hypotheses of the generalized concentration-compactness alternative I. Since $u_{\varepsilon}$ is almost extremal we have $\nu(\bar{\Omega})=S^{F}$ and it suffices to exclude compactness. Suppose on the contrary that compactness occurs. Then $v_{\varepsilon} \rightarrow v_{0} \neq 0$ in $L^{2^{*}}(\Omega)$ and a.e. Moreover $S^{F} \leq F_{0}^{+} S^{*}$. Thus compactness is excluded if $F_{0}^{+}<S^{F} / S^{*}$. We may assume $F_{0}^{+}>0$ since otherwise $S^{F}=0$ which contradicts the hypothesis $F \not \equiv 0$. The assertion in the case $F_{0}^{-}=F_{0}^{+}$follows from the last claim of Theorem 12. As to the assertion for domains of finite volume we first consider the case $u_{\varepsilon} \geq 0$. Fix $t>0$ and let $r_{\varepsilon}(t)$ be the radius of the ball $\left\{v_{\varepsilon}{ }^{*}>t\right\}$ where ${ }^{*}$ denotes Schwarz symmetrization. Define $\bar{v}_{\varepsilon}=v_{\varepsilon}^{*}$ on $\left\{v_{\varepsilon}{ }^{*}>t\right\}$ and extend it to all of $\mathbb{R}^{\mathrm{n}}$ by the radial harmonic function vanishing at infinity. By (6) we have

$$
\int_{\mathbb{R}^{n}}\left|\nabla \bar{v}_{\varepsilon}\right|^{2}=\int_{\left\{v_{\varepsilon}^{*}>t\right\}}\left|\nabla v_{\varepsilon}^{*}\right|^{2}+t^{2}(n-2)\left|S^{n-1}\right| r_{\varepsilon}(t)^{n-2} .
$$

Since $\left\|\nabla v_{\varepsilon}^{*}\right\|_{2} \leq\left\|\nabla v_{\varepsilon}\right\|_{2} \leq 1$ we we can estimate the first part of the Dirichlet integral by

$$
\begin{aligned}
\int_{\left\{v_{\varepsilon}^{*}>t\right\}}\left|\nabla v_{\varepsilon}^{*}\right|^{2} & \leq 1-\int_{\left\{v_{\varepsilon}^{*} \leq t\right\}}\left|\nabla v_{\varepsilon}^{*}\right|^{2} \\
& \leq 1-t^{2}(n-2)\left|S^{n-1}\right| \frac{r_{\varepsilon}(t)^{n-2}}{1-\left(r_{\varepsilon}(t) / r_{\varepsilon}(0)\right)^{n-2}} .
\end{aligned}
$$

Let $R$ denote the radius of the ball $\Omega^{*}$ which is finite by hypothesis. Define $r(t):=\liminf _{\varepsilon \rightarrow 0} r_{\varepsilon}(t)$. Clearly $r_{\varepsilon}(0) \leq R$. Combining the above estimates and monotonicity of the function

$$
r \mapsto \frac{r^{2 n-4}}{R^{n-2}-r^{n-2}}
$$

we obtain

$$
\limsup _{\varepsilon \rightarrow 0} \int_{\mathbb{R}^{n}}\left|\nabla \bar{v}_{\varepsilon}\right|^{2} \leq 1-t^{2}(n-2)\left|S^{n-1}\right| \frac{r(t)^{2 n-4}}{R^{n-2}-r(t)^{n-2}} .
$$


The functional changes according to

$$
\begin{aligned}
\int_{\mathbb{R}^{n}} \varepsilon^{-2^{*}} F\left(\varepsilon \bar{v}_{\varepsilon}\right) & \geq \int_{\left\{v_{\varepsilon}^{*}>t\right\}} \varepsilon^{-2^{*}} F\left(\varepsilon v_{\varepsilon}^{*}\right) \\
& =\int_{\Omega} \varepsilon^{-2^{*}} F\left(\varepsilon v_{\varepsilon}\right)-\int_{\left\{v_{\varepsilon} \leq t\right\}} \varepsilon^{-2^{*}} F\left(\varepsilon v_{\varepsilon}\right) \\
& \geq S^{F}+o\left(\varepsilon^{0}\right)-c \int_{\left\{v_{\varepsilon} \leq t\right\}}\left|v_{\varepsilon}\right|^{2^{*}} \\
& \geq S^{F}-c \int_{\left\{v_{0} \leq t\right\}}\left|v_{0}\right|^{2^{*}}+o\left(\varepsilon^{0}\right)
\end{aligned}
$$

For small $t$ we have $r(t) \leq c>0$ because $v_{0} \neq 0$ and

$$
\left|\left\{v_{0}>t\right\}\right| \geq \liminf _{\varepsilon \rightarrow 0}\left|\left\{v_{\varepsilon} \geq t\right\}\right|
$$

As $\varepsilon \rightarrow 0$ we obtain by the generalized Sobolev inequality (5)

$$
\begin{aligned}
S^{F}-c|\Omega| t^{2^{*}} & \leq \limsup _{\varepsilon \rightarrow 0} \int_{\mathbb{R}^{\mathrm{n}}} \varepsilon^{-2^{*}} F\left(\varepsilon \bar{v}_{\varepsilon}\right) \\
& \leq S^{F}\left(1-t^{2}(n-2)\left|S^{n-1}\right| \frac{r(t)^{2 n-4}}{R^{n-2}-r(t)^{n-2}}\right)^{\frac{n}{n-2}} \\
& \leq S^{F}-\frac{C t^{2}}{R^{n-2}-r(t)^{n-2}}
\end{aligned}
$$

For small $t$ this is a contradiction. If $v_{\varepsilon}$ changes sign we symmetrize the positive and the negative part separately and use the relations

$$
\begin{aligned}
\int_{\mathbb{R}^{\mathrm{n}}}\left|\nabla u_{+}^{*}\right|^{2}+\int_{\mathbb{R}^{\mathrm{n}}}\left|\nabla u_{-}^{*}\right|^{2} & \leq \int_{\mathbb{R}^{\mathrm{n}}}|\nabla u|^{2}, \\
\int_{\mathbb{R}^{\mathrm{n}}} F\left(u_{+}^{*}\right)+\int_{\mathbb{R}^{\mathrm{n}}} F\left(u_{-}^{*}\right) & =\int_{\mathbb{R}^{\mathrm{n}}} F(u) .
\end{aligned}
$$

\subsection{Blow up and convergence to an entire extremal}

Although part 2 of Theorem 3 is almost identical with Theorem I.5 of Lions [10] we present a new proof based on the local generalized Sobolev inequality. It also applies to discontinuous integrands not covered by Lions' result. Note that $\left\|\nabla w_{\varepsilon}\right\|_{2} \leq 1$ and $\int_{\mathbb{R}^{n}} F\left(w_{\varepsilon}\right)=\int_{\Omega} F\left(u_{\varepsilon}\right) / \varepsilon^{2^{*}} \rightarrow S^{F}$. As Lions [10] we first apply the compactness-splitting-vanishing alternative (Lemma 23 below) to the measures

$$
\sigma_{\varepsilon}:=F\left(\tilde{w}_{\varepsilon}\right) d y \text { with } \tilde{w}_{\varepsilon}(y):=u_{\varepsilon}\left(\varepsilon^{\frac{2}{n-2}} y\right)
$$


Lemma 23 (P. L. Lions [9, p. 115]). - Every sequence $\left(\sigma_{\varepsilon}\right)$ of positive Borel measures on $\mathbb{R}^{\mathrm{n}}$ with

$$
\sigma_{\varepsilon}\left(\mathbb{R}^{\mathrm{n}}\right) \rightarrow S>0 \text { as } \varepsilon \rightarrow 0
$$

has a subsequence for which one of the following statements holds true.

1. Compactness: The measures $\left(\sigma_{\varepsilon}\right)$ converge up to translation, i.e. there is a sequence of centers $\left(x_{\varepsilon}\right)$ in $\mathbb{R}^{\mathrm{n}}$ such that for every $\delta>0$ a radius $R$ exists for which

$$
\sigma_{\varepsilon}\left(B_{x_{\varepsilon}}^{R}\right)>S-\delta
$$

for every $\varepsilon$.

2. Splitting: The measures $\left(\sigma_{\varepsilon}\right)$ separate into two distant pieces, i.e. there are $S_{1}, S_{2}>0$ with $S_{1}+S_{2}=S$, such that for every $\delta>0$ a radius $r$ and centers $\left(x_{\varepsilon}\right)$ exist, such that for every $R>r$

$$
\begin{aligned}
\sigma_{\varepsilon}\left(B_{x_{\varepsilon}}^{r}\right) & \geq S_{1}-\delta, \\
\sigma_{\varepsilon}\left(\mathbb{R}^{\mathrm{n}} \backslash B_{x_{\varepsilon}}^{R}\right) & \geq S_{2}-\delta
\end{aligned}
$$

for $\varepsilon$ small enough.

3. Vanishing: The measures $\left(\sigma_{\varepsilon}\right)$ smear out in the sense that for every radius $R$ one has

$$
\lim _{\varepsilon \rightarrow 0} \sup _{x \in \mathbb{R}^{n}} \sigma_{\varepsilon}\left(B_{x}^{R}\right)=0 .
$$

As to the proof of part 2 of Theorem 3 we first prove compactness in the above sense by exclusion of splitting and vanishing. Then we apply the generalized concentration-compactness alternative II to obtain convergence in the $D^{1,2}$ sense. This requires exclusion of concentration. The condition $F_{0}^{+}<S^{F} / S^{*}$ prevents the sequence from vanishing, the condition $F_{\infty}^{+}<S^{F} / S^{*}$ excludes concentration. With no loss of generality we can assume $x_{\varepsilon}=0$.

STEP 24. - Exclusion of splitting.

Proof. - Suppose for a contradiction that there are $S_{1}, S_{2}>0$ with $S_{1}+S_{2}=S^{F}$, such that for every $\delta>0$ a radius $r$ exists, such that

$$
\begin{aligned}
S_{1}-\delta & \leq \int_{B_{0}^{r}} F\left(\tilde{w}_{\varepsilon}\right), \\
S_{2}-\delta & \leq \int_{\mathbb{R}^{\mathrm{n}} \backslash B_{0}^{R}} F\left(\tilde{w}_{\varepsilon}\right)
\end{aligned}
$$


for all $R>r$ and $\varepsilon$ small enough. For every $\delta^{\prime}>0$ the local generalized Sobolev inequality (Corollary 9) provides two radii $R, \rho$ such that $R>\rho>r$ and

$$
\begin{aligned}
& S_{1}-\delta \leq \int_{B_{0}^{r}} F\left(\tilde{w}_{\varepsilon}\right) \leq S^{F}\left(\int_{B_{0}^{\rho}}\left|\nabla \tilde{w}_{\varepsilon}\right|^{2}+\delta^{\prime}\right)^{\frac{n}{n-2}}, \\
& S_{2}-\delta \leq \int_{\mathbb{R}^{n} \backslash B_{0}^{R}} F\left(\tilde{w}_{\varepsilon}\right) \leq S^{F}\left(\int_{\mathbb{R}^{n} \backslash B_{0}^{\rho}}\left|\nabla \tilde{w}_{\varepsilon}\right|^{2}+\delta^{\prime}\right)^{\frac{n}{n-2}} .
\end{aligned}
$$

Adding these inequalities we obtain

$$
\left(\frac{S_{1}-\delta}{S^{F}}\right)^{\frac{n-2}{n}}+\left(\frac{S_{2}-\delta}{S^{F^{\prime}}}\right)^{\frac{n-2}{n}} \leq 1+2 \delta^{\prime}
$$

For $\delta^{\prime}$ and $\delta$ small enough this is a contradiction since $S_{1}>0$ and $S_{2}=S^{F}-S_{1}>0$.

STEP 25. - Exclusion of vanishing.

Proof. - Suppose on the contrary that

$$
\sup _{R>0} \lim _{\varepsilon \rightarrow 0} \sup _{x \in \mathbb{R}^{n}} \int_{B_{x}^{R}} F\left(\tilde{w}_{\varepsilon}\right)=0 .
$$

By the local generalized Sobolev inequality and the fact that $u_{\varepsilon}$ is almost extremal the above assumption implies

$$
\begin{aligned}
S^{F} & =\inf _{R>0} \lim _{\varepsilon \rightarrow 0} \inf _{x \in \mathbb{R}^{\mathrm{n}}} \int_{\mathbb{R}^{\mathrm{n}} \backslash B_{x}^{R}} F\left(\tilde{w}_{\varepsilon}\right) \\
& \leq \inf _{r>0} \lim _{\varepsilon \rightarrow 0} \inf _{x \in \mathbb{R}^{\mathrm{n}}} S^{F}\left(1-\int_{B_{x}^{r}}\left|\nabla \tilde{w}_{\varepsilon}\right|^{2}\right)^{\frac{n}{n-2}}
\end{aligned}
$$

or

$$
\sup _{r>0} \lim _{\varepsilon \rightarrow 0} \sup _{x \in \mathbb{R}^{n}} \int_{B_{x}^{r}}\left|\nabla \tilde{w}_{\varepsilon}\right|^{2}=0 .
$$

Another application of the local generalized Sobolev inequality yields vanishing with respect to a modified volume integrand, namely

$$
\lim _{\varepsilon \rightarrow 0} \sup _{x \in \mathbb{R}^{n}}\left|\left\{\left|\tilde{w}_{\varepsilon}\right| \geq \delta\right\} \cap B_{x}^{r}\right|=0 \text { for every } \delta>0, r>0 .
$$


By hypothesis there exists a $t_{0}>0$ such that

$$
\sup _{|t| \leq t_{0}} \frac{F(t)}{|t|^{2^{*}}}<\frac{S^{F}}{S^{*}}
$$

Define

$$
F_{1}(t):=\left\{\begin{array}{ll}
F(t) & \left(|t|<t_{0}\right), \\
0 & \left(|t| \geq t_{0}\right),
\end{array} \quad F_{2}:=F-F_{1}\right.
$$

Then

$$
F_{2}(t) \leq F_{3}(t):=c\left(|t|-\frac{t_{0}}{2}\right)_{+}^{2^{*}}
$$

We claim that

$$
\lim _{\varepsilon \rightarrow 0} \int_{\mathbb{R}^{\mathrm{n}}} F_{2}\left(\tilde{w}_{\varepsilon}\right)=0
$$

To this end consider a cube $Q_{z}:=z+[0,1)^{n}$ with $z \in Z^{n}$ and observe that (13) implies

$$
\lim _{\varepsilon \rightarrow 0} \sup _{z \in Z^{n}}\left|\left\{\left|\tilde{w}_{\varepsilon}\right|>\frac{t_{0}}{2}\right\} \cap Q_{z}\right|=0
$$

i.e. that $F_{3}\left(\tilde{w}_{\varepsilon}\right)$ vanishes on most of $Q_{z}$. Application of Lemma 28 from the appendix yields

$$
\int_{Q_{z}} F_{3}\left(\tilde{w}_{\varepsilon}\right) \leq c\left(\int_{Q_{z}}\left|\nabla \tilde{w}_{\varepsilon}\right|^{2}\right)^{\frac{n}{n-2}}
$$

for $\varepsilon$ small with a constant $c$ independent of $z$. After summation over $z \in Z^{n}$ we find

$$
\begin{aligned}
\int_{\mathbb{R}^{\mathrm{n}}} F_{2}\left(\tilde{w}_{\varepsilon}\right) & \leq \int_{\mathbb{R}^{\mathbf{n}}} F_{3}\left(\tilde{w}_{\varepsilon}\right) \leq c \sum_{z \in Z^{n}}\left(\int_{Q_{z}}\left|\nabla \tilde{w}_{\varepsilon}\right|^{2}\right)^{\frac{n}{n-2}} \\
& \leq c \sup _{z \in Z^{n}}\left(\int_{Q_{z}}\left|\nabla \tilde{w}_{\varepsilon}\right|^{2}\right)^{\frac{2}{n-2}} \int_{\mathbb{R}^{\mathbf{n}}}\left|\nabla \tilde{w}_{\varepsilon}\right|^{2}
\end{aligned}
$$

Now (15) follows from (12). In view of (14) we obtain

$$
S^{F}=\limsup _{\varepsilon \rightarrow 0} \int_{\mathbb{R}^{n}} F\left(\tilde{w}_{\varepsilon}\right)=\limsup _{\varepsilon \rightarrow 0} \int_{\mathbb{R}^{n}} F_{1}\left(\tilde{w}_{\varepsilon}\right)<S^{F}
$$

which is a contradiction. 
STEP 26. - Exclusion of concentration; convergence of $\left(w_{\varepsilon}\right)$ in $D^{1,2}\left(\mathbb{R}^{\mathrm{n}}\right)$ to an entire extremal.

Proof. - By Lemma 23 and the Steps 24 and 25 there is a sequence of points $\left(x_{\varepsilon}\right)$ such that the measures $F\left(w_{\varepsilon}\right) d y$ do not concentrate at infinity. A subsequence of $\left(w_{\varepsilon}\right)$ satisfies the hypotheses of Theorem 20 on $\mathbb{R}^{\mathrm{n}}$ with $\nu\left(\mathbb{R}^{\mathrm{n}}\right)=S^{F}$. Compactness in the sense of the compactness-splittingvanishing alternative excludes concentration at infinity. Concentration at a finite point is excluded by our growth condition at infinity. Indeed, if $\left(w_{\varepsilon}\right)$ concentrates at a finite point then

$$
\begin{aligned}
S^{F^{\prime}} & =\lim _{\varepsilon \rightarrow 0} \int_{\left\{\left|w_{\varepsilon}\right| \geq t\right\}} F\left(w_{\varepsilon}\right) \\
& \leq\left(F_{\infty}^{+}+o(1)\right) S^{*}<S^{F} \text { as } t \rightarrow \infty .
\end{aligned}
$$

Thus alternative (b) in part two of Theorem 20 occurs.

STEP 27. $-x_{\varepsilon} \rightarrow x_{0}$.

Proof. - Let $v_{\varepsilon}=u_{\varepsilon} / \varepsilon$. By part 1 of Theorem 3 there exists a subsequence and $x_{0} \in \bar{\Omega}$ such that $\left|\nabla v_{\varepsilon}\right|^{2} d x \rightarrow^{*} \delta_{x_{0}}$ in $\mathcal{M}(\bar{\Omega})$. On the other hand one has

$$
0<\int_{B_{0}^{R}}|\nabla w|^{2}=\lim _{\varepsilon \rightarrow 0} \int_{B_{0}^{R}}\left|\nabla w_{\varepsilon}\right|^{2}=\lim _{\varepsilon \rightarrow 0} \int_{B_{x_{\varepsilon}}^{R_{\varepsilon}}}\left|\nabla v_{\varepsilon}\right|^{2}
$$

for $R$ large enough with $R_{\varepsilon}:=\varepsilon^{\frac{2}{n-2}} R$. This inequality fails if $x_{\varepsilon}$ does not tend to $x_{0}$.

\section{APPENDIX}

In the proof of part 2 of Theorem 3 we used the following variant of the standard Poincaré-Sobolev inequality. The corresponding variant of Poincaré's inequality can be found in Morrey's book [11, Theorem 3.6.5].

LEMma 28. - Suppose $Q$ is a connected domain of finite volume with Lipschitz boundary and let $\theta \in[0,1)$. Then there is a constant $c(Q, \theta)$ such that

$$
\|w\|_{2^{*}} \leq c(Q, \theta)\|\nabla w\|_{2}
$$

for every $w \in H^{1}(Q)$ with $|\{w \neq 0\}| \leq \theta|Q|$. 
Proof. - Suppose the assertion was false. Then there exists a sequence $\left(w_{k}\right)$ such that $\left\|w_{k}\right\|_{2^{*}}=1,\left|\left\{w_{k} \neq 0\right\}\right| \leq \theta|Q|$ and $\left\|\nabla w_{k}\right\|_{2} \rightarrow 0$. By Rellich's theorem $w_{k} \rightarrow w$ in $L^{2}(Q)$ for a subsequence. Hence $w_{k} \rightarrow w$ in $H^{1}(Q)$ and $L^{2^{*}}(Q)$. Moreover $\nabla w=0$ a.e. By connectedness of $Q$ the limit function is a constant, say $w=t \in \mathbb{R}$ a.e. By the normalization of $w_{k}$ and strong $L^{2^{*}}$ convergence we deduce $t \neq 0$. On the other hand $\left|\left\{\left|w_{k}-t\right|>\delta\right\}\right| \rightarrow 0$ as $k \rightarrow \infty$. For $\delta<|t|$ this contradicts $\left|\left\{w_{k} \neq 0\right\}\right| \leq \theta|Q|$.

\section{REFERENCES}

[1] C. BANDlE and M. FluChER, Harmonic radius and concentration of energy; hyperbolic radius and Liouville's equations $\Delta U=e^{U}$ and $\Delta U=U^{(n+2) /(n-2)}$. SIAM Rev., Vol. 38, 2, 1996, pp. 191-238.

[2] C. Dellacherie and P.-A. Meyer, Probabilities and potential. Vol. 29 of North-Holland Mathematics Studies. North-Holland Publishing Co., Amsterdam, 1978.

[3] M. Filrehfr, An asymptotic formula for the minimal capacity among sets of equal area. Calc. Var. Partial Differential Equations, Vol. 1, 1, 1993, pp. 71-86.

[4] M. FluChER and S. MüLlER, Radial symmetry and decay rate of variational ground states in the zero mass case. SIAM J. Math. Anal., Vol. 29, 3, 1998, pp. 712-719

[5] M. FluCher, A. GaRroni and S. MÜlLeR, Concentration of low energy extremals: Identification of concentration points. (In preparation).

[6] M. FLUCHER and M. RUMPF, Bernoulli's free-boundary problem, qualitative theory and numerical approximation. J. Reine Angew. Math., Vol. 486, 1997, pp. 165-204.

[7] M. FluCHER and J. WEI, Asymptotic shape and location of small cores in elliptic free-boundary problems. Math. Z., Vol. 228, 1998, pp. 683-703.

[8] P. M. Gruber and J. M. Wills Eds. Handbook of convex geometry., Vol. B. North-Holland Publishing Co., Amsterdam, 1993.

[9] P.-L. Lions, The concentration-compactness principle in the calculus of variations. The locally compact case. I. Ann. Inst. H. Poincaré Anal. Non Linéaire, Vol. 1, 2, 1984 , pp. 109-145.

[10] P.-L. Lions, The concentration-compactness principle in the calculus of variations. The limit case. I. Rev. Mat. Iberoamericana, Vol. 1, 1, 1985, pp. 145-201.

[11] Charles B. MoRreY, Multiple integrals in the calculus of variations. Springer-Verlag New York, Inc., New York, 1966. Die Grundlehren der mathematischen Wissenschaften, Band 130.

[12] G. Talenti, Elliptic equations and rearrangements. Ann. Scuola Norm. Sup. Pisa Cl. Sci., Vol. 4, 3, 4, 1976, pp. 697-718.

(Manuscript received February 24, 1997.) 